\title{
Green Sample Preparation Techniques for Chromatographic Determination of Small Organic Compounds
}

\author{
Jacek Namieśnik, Agata Spietelun, and Łukasz Marcinkowski
}

\begin{abstract}
Accurate monitoring of the state of the environment requires the introduction of additional isolation and/or preconcentration step prior to the chromatographic determination. In this work the information about novel methodological and instrumental solutions in relation to different variants of microextraction techniques are presented. Attention is focused on analytical procedure and suitable analytical tools for extraction of the analytes from samples with a complex matrix composition and on minimazing of organic solvent consumption. The described modifications of extraction techniques deal with system miniaturization and/or automation, the use of additional agents to enhance the effectiveness of extraction like ultrasound and physical agitation, and electrochemical methods. Particular attention was given to pro-ecological aspects therefore the possible use of novel, non-toxic extracting agents and selective sorbents have been evaluated in depth.
\end{abstract}

Index Terms-Extraction techniques, sample preparation, solid phase microextraction, liquid phase extraction, green analytical chemistry.

\section{INTRODUCTION}

One of the most important challenges facing contemporary analytical chemistry is the determination of a wide range of analytes in samples of media with a complex matrix composition. It has also become imperative that new analytical methodologies should comply with the principles of sustainable development and green chemistry. According to the most popular definition of green chemistry more attention should be paid to the problem of the preservation of natural resources and to the impact of human activities on the environment. This new green approach of analytical chemists to their work is also described in literature as the three R's, which stands for replace, reduce and recycle (replacement of toxic solvents with green solvents, reduction of solvent consumption and waste production, and solvent recycling) [1] Milestones of green chemistry are presented below:

1) 1987 - report of the World Commission on Environment and Development (the idea of sustainable development considered relationship between economic growth and caring for the environment and health of the present and future generations)

2) 1990 - application of the green chemistry rules passed by the US Congress (the implementation of sustainable

Manuscript received July 10, 2014; revised September 29, 2014

The authors are with the Gdańsk University of Technology, Faculty of Chemistry, Department of Analytical Chemistry, Gdańsk, Poland (e-mail: chemanal@pg.gda.pl). development in the chemical laboratory and industrial scale)

3) 1991 - introduction of the term green chemistry by P. Anastas within the framework of the US Environmental Protection Agency program

4) 1993 - establishment of the comprehensive US Green Chemistry Program

5) 1997 - description of implementation of green chemistry rules in analytical chemistry

6) 1999 - introduction of the term Green Analytical Chemistry.

The principles of green analytical chemistry are implemented at each stage of analytical procedure [2] (Fig. 1)

The use of ultrasonic irradiation, microwaves, electrochemical support and green, non-toxic extraction medium (e.g. ionic liquids, supercritical liquids, surfactant solutions, supramolecular solvents) and commercial autosampler, resulted in the introduction of fast, low cost, automated processes which integrate of sample pretreatments and analytical measurements and resulted in improving of the available methodologies. Liquid phase microextraction techniques wherein the amount of organic solvents used during the extraction process were reduced, resulted in lower costs of waste treatment, and has become one of the most popular research topics in green analytical chemistry. A lot of review papers about green analytical chemistry rules and the implementation of them at each stage of analytical procedure were published [2]-[5].

Suitable monitoring of compounds presented in samples at trace levels usually requires a preliminary step of isolation/enrichment of analytes because most of analytical techniques are not sensitive enough for direct determination of trace compounds; moreover, in many cases, the analytes have to be separated from the matrix before the analysis.

The sample preparation step is of fundamental importance for the accuracy and reliability of the final analysis results. In the context of the requirements of green chemistry less timeand labour-consuming techniques, especially solventless sample preparation techniques are gaining particular importance. This group of sample pretreatment techniques includes extraction techniques using a carrier gas, solid sorbents combined with thermal desorption of retained analytes, and techniques using safe, non-toxic extraction media and green solvents.

Extraction techniques rely primarily on the enrichment of an analyte to a concentration above the limit of detection if the instrument used, also enable interferents to be removed during measurement and isolation of analytes from sample 
matrix and/or matrix simplification [1], [5]-[7].

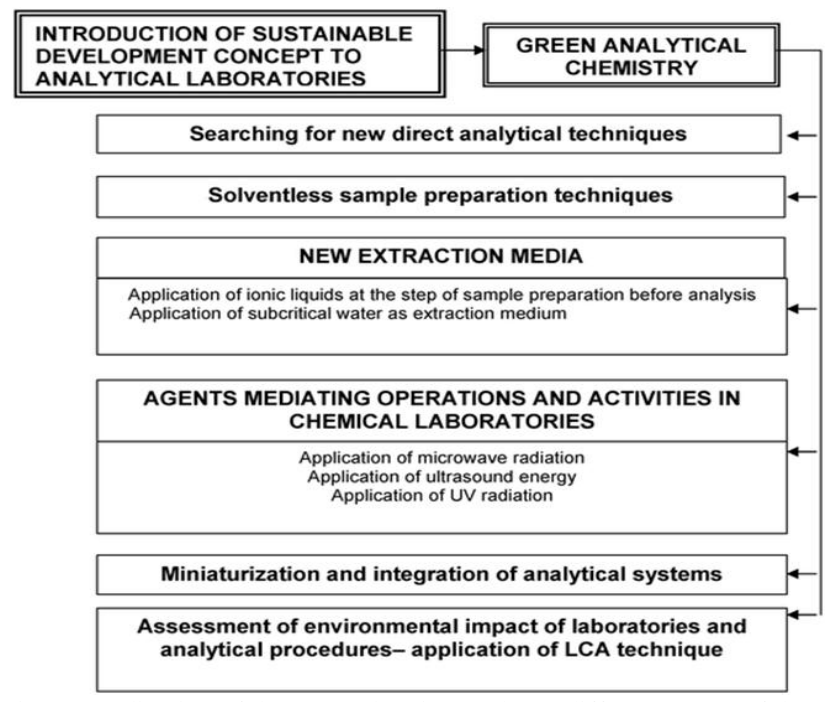

Fig. 1. Application of the green chemistry rules at different stages of the analytical procedure [2].

Microextraction techniques find increasing applications in the sample preparation step before chromatographic determination of analytes in samples with a complex composition. These methodological solutions together with properly matched sampling devices for collecting analytes, enable to reduce the number of errors during the sample pretreatment prior to chromatographic analysis as well as to limit the negative impact of this analytical step on the environment and the health of laboratory employees.

\section{NOVEl SOlutions IN THE FIELD OF SOlID-PHASE MICROEXTRACTION TECHNIQUE}

Solid-phase microextraction (SPME) is one of the most popular green, solventless techniques used for sample preparation applied in analytical practice for isolation/preconcentration of a wide range of analytes present in media with complex matrix composition [8]. An SPME device consists basically of a silica fibre or metal core coated with a thin layer of a suitable polymeric sorbent, fixed within the needle of a syringe-like arrangement. Extraction of analytes is performed by immersing the fibre in gaseous or liquid medium; analytes can also be extracted from the headspace above the sample. After extraction the fibre is placed directly in the injector of the monitoring instrument, where desorption of the analytes retained on the fibre occurs [1].

Due to the possible use of the SPME technique for extraction of analytes presented at trace levels, a number of studies have been undertaken to improve the extraction efficiency of this method [1], [9] (Fig. 2).

To this end, novel design solutions were developed, e.g.

1) internally cooled fiber and its automated and miniaturized version

2) in-tube SPME (devices which contain the stationary phase as internal coating of the needle or the capillary wall) automation of SPME device in various configuration
3) liquid-liquid-solid microextraction and its dynamic version (molecularly imprinted polymer coated fiber is placed inside the porous polypropylene membrane filled with an organic solvent or fiber is immersed in an organic solvent interfaced with aqueous phase)

4) electrosorption-enhanced SPME and its modificationelectrochemical solid-phase nanoextraction (electrochemically aided variant of SPME technique, wherein the fiber plays a role of the working electrode; by applying a negative potential to the SPME fiber, the coating becomes negatively charged and an electric field is formed, where positively charged analytes are attracted to the fiber surface and become retained in the coating layer via complementary charge attraction)

5) electromembrane surrounded SPME (analytes migrated through the liquid membrane to the aqueous acceptor phase and then they were adsorbed on the SPME fibre, which acts as the cathode)

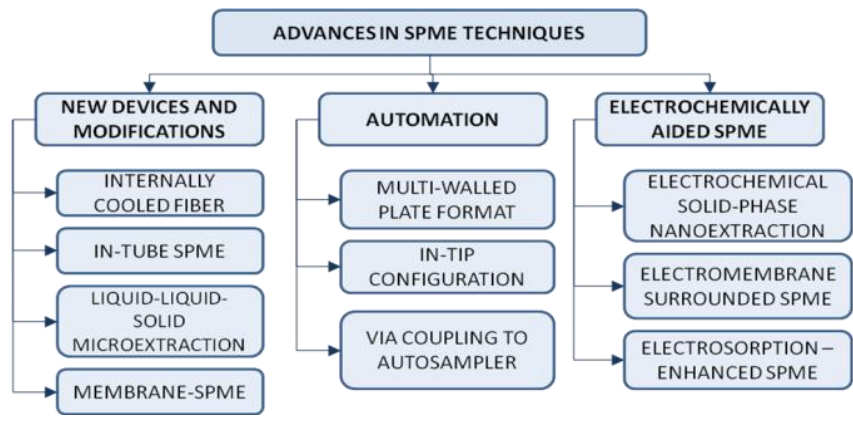

Fig. 2. Trends in SPME technique [1]

In 2009, a novel procedure for the SPME fiber preparation was proposed which involved a physical separation of extraction medium from the sample by means of a membrane (Membrane solid-phase microextraction). According to this concept, a layer of sorbent material is deposited onto silica fiber was covered with a hydrophobic, and thermally-stable external layer of poly(dimethylsiloxane), which serves as a membrane, to avoid of dissolution of extraction medium in the sample. Pseudo-liquids, i.e. high density polymers which resemble viscous fluid are used as an extrahent in this two-phase sorption system. The analytes are retained on the fiber via dissolution in the extraction medium [10], [11].

Most commonly published studies on the SPME development relate to the use of completely new materials as an extraction phase such as [1], [9], [12]:

1) conductive polymers

2) molecularly imprinted polymers

3) metal complex imprinted polymers

4) materials obtained via the sol-gel process

5) multi-walled and single-walled carbon nanotubes, and their nanocomposite with polypyrrole/polyaniline

6) carbon materials e.g. fullerol, carbon aerogels and wormhole-like mesoporous carbons, graphene, nanoporous carbon, ceramic carbon materials

7) mesoporous and nanoporous silicates, and aniline-silica nanocomposite, flower-like silica microstructures

8) metal nanoparticles e.g. gold, silver, zinc oxide, titanium oxide

9) ionic liquids and polymeric ionic liquids. 


\section{NOVEL METHODOLOGICAL SOLUTIONS IN THE FIELD OF STIRRING DEVICES FOR SOLID PHASE MICROEXTRACTION}

Stir bar sorptive extraction (SBSE) technique was introduced in 1999 [13] and since then, has been increasingly applied to determine contaminants in environmental, biological and food samples. An SBSE device consists basically of a 1-4 cm long magnetic stirrer is coated with poly(dimethylsiloxane). During the extraction, the stir bar is immersed in the sample solution or hanging in the headspace above the sample. The analytes retained by the sorptive element are desorbed via thermal or liquid desorption. The automation of the SBSE technique is possible, and multi-shot mode has been introduced. Most commonly studies on the SBSE development deal with the use of new materials used for coating of magnetic stirrers [1] e.g.:

1) poly(ethylene glycol) modified silicone

2) polyacrylate

3) polyurethane foams

4) poly(phthalazine ether sulfone ketone)

5) restricted access materials

6) silicon materials

7) sorbents obtained with sol-gel techniques

8) cyclodextrine

9) polyvinyl alcohol

10) carbon nanotubes- poly(dimethylsiloxane)

11) poly(dimethylsiloxane)/polypyrrole

12) molecularly imprinted polymers

13) monolithic materials.

In recent years, additional efforts have been made to improve the solid phase microextraction through the development of simple and original device which also serves as a magnetic stirrer [1]. An innovative technique called adsorptive $\mu$-extraction and its two versions, i.e. bar adsorptive $\mu$-extraction and multi-spheres adsorptive $\mu$-extraction were elaborated. In the adsorptive $\mu$-extraction technique, fragments of polypropylene tube or polystyrene spheres are covered with powdered sorbent of varying polarity and porosity. Activated carbon and divinylbenzene mixed with polystyrene were the most commonly sorbent used. After the extraction, the analytes were desorbed into a small amount of solvent and determined by using gas- or liquid chromatography. In the technique proposed under the name of stir-rod-sorptive extraction device consists of a metallic wire with a magnet at one end to which a sorbent-coated glass ending is attached. In 2011, the first report about stir cake sorptive extraction appeared; the monolithic cake of sorbent was used as an extraction medium placed inside a special holder designed for this purpose; the holder plays a role of a magnetic stirrer which allows for performing the extraction of analytes and sample stirring at the same time. Monolithic discs are made of molecularly imprinted polymer and ionic liquid polymers could be also used in this solution. In the literature, there is also information on the technique known as rotating disk sorbent extraction. In this technique, the stationary phase is deposited on the Teflon disk, rotated with a magnetic stirrer. After the analyte extraction, the analytes are desorbed into a small volume of an organic solvent and determined by using gas- or liquid chromatography [1].

\section{Novel Solutions IN THE FIELD OF SINGLE-DroP MICROEXTRACTION TECHNIQUE}

One of the most popular techniques of liquid-phase microextraction, in which the use of solvents has been significantly reduced is a single-drop microextraction (SDME) [14]. In this technique, the extraction occurs via dissolution of analytes in a drop of solvent suspended at the end of a microsyringe needle which has been immersed in the sample or extraction could be done from a headspace above the sample. After extraction, the drop is withdrawn back into the syringe and then dispensed into a measuring device for final determination. Information about two other variants of SDME can be found in the published literature: drop to drop- SDME (analytes are extracted from the sample into a drop of solvent), and liquid-liquid-liquid microextraction (analytes are extracted from an aqueous phase into an organic phase, and then back-extracted into a drop of an aqueous phase) [5].

To increase the mass transfer, a dynamic version of SDME and its nano-scale variant, namely, dynamic liquid phase nanoextraction were also developed. In 2007, SDME was fully automated by coupling with commercially available autosampler and sequential injection system. The SDME technique was also applied in the miniaturized version of lab-on-a-chip, known as droplet-membrane-droplet liquid-phase microextraction, in which analytes are extracted through a supported liquid membrane into few microliters of an acceptor phase. Also electrochemically-assisted version coupled on-line with microchip capillary electrophoresis was also proposed [5].

In 2006, a new modification of the SDME techniques was proposed; a drop of water-insoluble solvent with a density lower than that of water was placed directly onto the surface of an aqueous sample. This technique knows as directly suspended droplet microextraction. Another modified version of the SDME technique using solvents less dense than water and melted at room temperature is known as solidification of a floating organic drop microextraction (SFOD). Here, a drop of extrahent is placed on the surface of an aqueous sample, and after the extraction, the vessel containing the sample is cooled on ice bath until the extraction medium solidifies. Next, the sorptive phase is transferred to another vessel in which it immediately melts. Liquid extraction medium is later dispended into a measuring instrument for the final determination of analytes [5].

Supramolecular assembly-based coacervates (e.g. surfactant micelles) due to their unique array of physicochemical properties are often applied in analytical techniques as extrahents. Vesicular-based coacervate was also used as solvent in SDME, this technique is known as single-drop coacervative microextraction, and its combination with SFOD technique is known as solidified floating vesicular coacervative drop microextraction. Also ionic liquids are often used in SDME technique because of to their high viscosity and surface tension which helps to form a stable drop [5]. 


\section{NOVEL SOLUTIONS IN THE FIELD OF HOLLOW FIBER LIQUID-PHASE MiCROEXTRACTION TECHNIQUE}

Liquid extrahent could be also placed inside a porous, semipermeable polymeric membrane tube, this technique is known as hollow fiber liquid-phase microextraction (HF-LPME) [15]. The tube is attached to the needle of a microsyringe and immersed in the sample or above it. After the extraction, the extraction phase is drawn inside the syringe and then injected into a measuring instrument for the determination of analytes. To this end, novel design solutions were developed [5] e.g.

1) hollow fiber membrane liquid-liquid-liquid microextraction (version also with ionic liquid)

2) solid phase membrane tip extraction (used carbon nanotubes enclosed in a cone shaped hollow fiber attached to a pipette tip)

3) solvent cooling assisted dynamic hollow-fiber-supported headspace liquid phase microextraction (cooled extrahent is pumped through a porous polymeric membrane)

4) automated, dynamic version of the HF-LPME technique by coupling with commercial autosampler

5) electrochemically aided methods known as electro membrane isolation and electro membrane extraction, and its nano-version (electrode is placed inside the membrane of a classical HF-LPME device)

6) miniaturized to the microchip level version of electro membrane extraction

By combining the advantages of membrane techniques and stirred devices for liquid-liquid microextraction, a new technique was proposed [5]:

1) solvent bar microextraction

2) stir membrane liquid-liquid microextraction

3) hollow fiber solid-liquid phase microextraction.

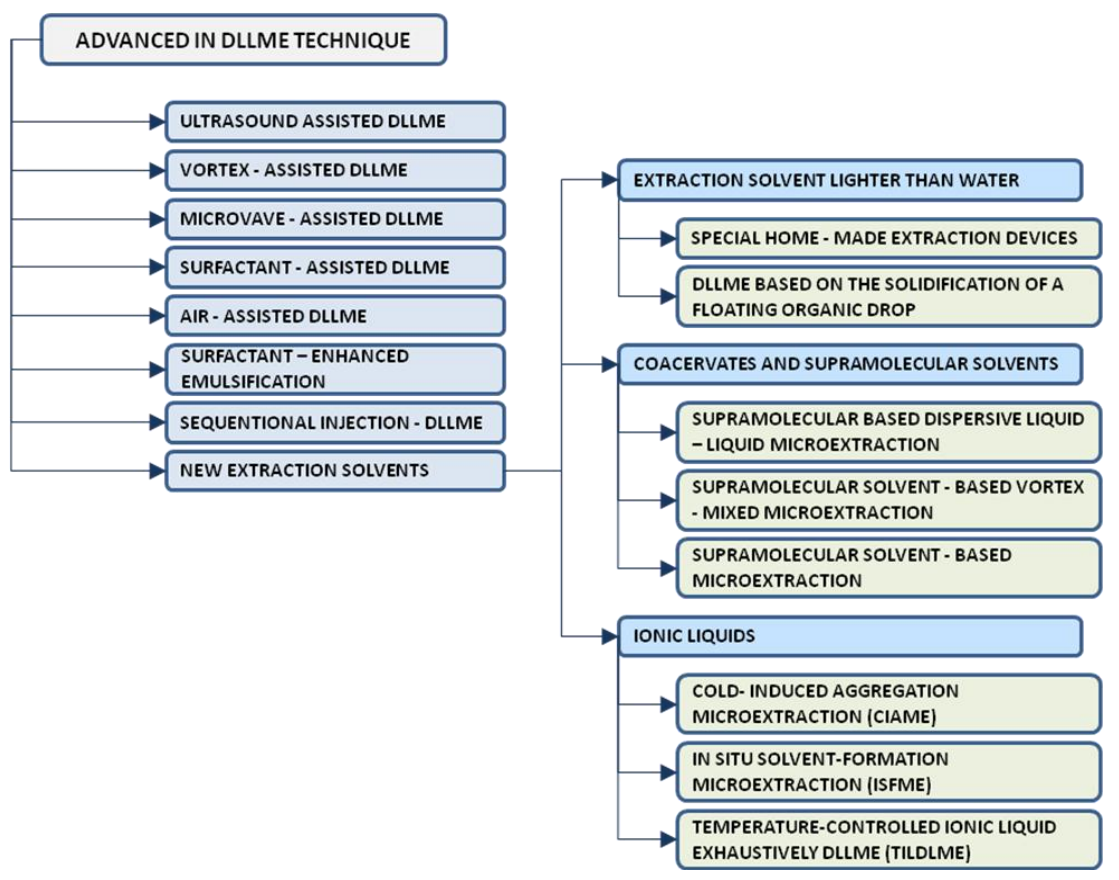

Fig. 3. Novel designs and innovative methodologies in DLLME technique [5].

\section{NOVEL SOLUTIONS IN THE FIELD OF DISPERSIVE LIQUID-LIQUID MICROEXTRACTION TECHNIQUE}

In 2006, a novel microextraction technique was reported which involved the extraction of analytes from aqueous samples into a small volume of organic solvent dispersed in the sample. In the original version of dispersive liquid-liquid microextraction (DLLME) the dispersion of the extrahent drops was achieved by using a third liquid phase which was immiscible with both, the sample and the extrahent, and served as dispersant [16]. After the extraction, samples are shaked and later centrifuged in order to separate the extraction phase. A fully-automated version of DLLME, named sequential injection dispersive liquid-liquid microextraction, is also available. A number of studies have been undertaken to improve the extraction efficiency of this method [5] (Fig. 3).

To eliminate toxic solvents, such as chlorinated hydrocarbons, alternative solvents can be applied in DLLME technique [5] e.g.:
1) solvents less dense than water (without the use of a dispersing agent and centrifugation step, performed in special extraction devices), most often used in combination with solidification of a floating organic drop

2) coacervates and supramolecular solvents (supramolecular based dispersive liquid-liquid microextraction, supramolecular solvent-based vortex-mixed microextraction, supramolecular solvent-based microextraction)

3) ionic liquid (in microwave-, ultrasound-, and vortex-assisted modes, in situ solvent-formation microextraction, temperature controlled ionic liquid dispersive liquid phase micro-extraction, cold-induced aggregation microextraction).

The application of a third, dispersion phase may decrease the value of the distribution ratio therefore, the increasing trend is to avoid the use of toxic dispersing solvent and to aid the process of dispersion/emulsification by [5]:

1) ultrasound treatment (ultrasound-assisted emulsification microextraction and its SFOD version, ultrasound 
dispersion liquid-liquid microextraction, ultrasound-assisted surfactant-enhanced emulsification microextraction and its SFOD version)

2) physical mixing by agitation (vortex-assisted liquid-liquid microextraction, vortex-assisted surfactant-enhanced emulsification liquid-liquid microextraction and its SFOD version)

3) sucking and injecting of the mixture of sample and extraction solvent with a syringe for several times (air-assisted liquid-liquid microextraction)

4) replacing of toxic dispersing solvent by a surfactant solution (surfactant-assisted dispersive liquid-liquid microextraction, ion pair based surfactant assisted microextraction).

\section{CONCLUSIONS}

Determination of analytes which are present in samples at a very low concentration level usually requires analytical procedures that include a preliminary step of the target analyte isolation/enrichment prior to the quantification step, because conventional multi-step procedures are not sensitive enough. Moreover the classical sorbents are characterized by low selectivity for target analytes, therefore the novel stationary phases were introduced e.g. polymeric ionic liquids, carbon nanotubes, silica and carbon sorbents, and molecularly imprinted polymers. Also novel green, non toxic solvents e.g. ionic liquids, supercritical liquids, and supramolecular solvents are used. A lot of novel modifications of well known techniques that are based on different approaches like chip-level miniaturization, automation; the use of ultrasounds, mechanical agitation, and electrochemical procedures; or the solidification of extrahent was introduced. The application of green microextraction techniques in the preliminary steps of chromatographic analysis enables the reduction of errors originated during sample preparation as well as limits the negative impact of harmful vapors originating from organic solvents on the environment and the health of laboratory personnel.

\section{ACKNOWLEDGEMENTS}

The project was funded by the Polish National Science Centre grant No. DEC-2011/01/N/ST4/01941

\section{ABBREVIATIONS AND ACRONYMS}

DLLME: dispersive liquid-liquid microextraction

HF-LPME: hollow fiber liquid-phase microextraction

SBSE: stir bar sorptive extraction

SDME: single drop microextraction

SFOD: solidification of a floating organic drop

SPME: solid-phase microextraction

\section{REFERENCES}

[1] A. Spietelun, Ł. Marcinkowski, M. de la Guardia, and J. Namieśnik, "Recent developments and future trends in solid phase microextraction techniques towards green analytical chemistry," J. Chromatogr. A, vol. 1321, pp. 1-13, Dec. 2013.

[2] M. Tobiszewski, A. Mechlińska, and J. Namieśnik, "Green analytical chemistry- theory and practice," Chem. Soc. Rev., vol. 39, pp. 2869-2878, Jan 2010.
[3] M. de la Guardia and S. Garrigues, Challenges in Green Analytical Chemistry, 1st ed., Cambridge, U.K., Royal Society of Chemistry, 2011.

[4] A. Gałuszka, Z. Migaszewski, and J. Namieśnik, "The 12 principles of green analytical chemistry and the SIGNIFICANCE mnemonic of green analytical practices," Trends Anal. Chem., vol. 50, pp. 78-84, October 2013.

[5] A. Spietelun, Ł. Marcinkowski, M. de la Guardia, and J. Namieśnik, "Green aspects, developments and perspectives of liquid phase microextraction techniques," Talanta, vol. 119, pp. 34-45, Feb. 2014

[6] M. Tobiszewski, A. Mechlińska, B. Zygmunt, and J. Namieśnik, "Green analytical chemistry in sample preparation for determination of trace organic pollutants," Trends Anal. Chem., vol. 28, pp. 943-951, Sept. 2009.

[7] W. Wardencki, J. Curyło, and J. Namieśnik, “Trends in solventless sample preparation techniques for environmental analysis," Biochem. Biophys. Methods, vol. 70, pp. 275-288, March 2007.

[8] C. L. Arthur and J. Pawliszyn, "Solid phase microextraction with thermal desorption using fused silica optical fibers," Anal. Chem., vol. 62, pp. 2145-2148, Oct. 1990.

[9] A. Spietelun, A. Kloskowski, W. Chrzanowski, and J. Namieśnik, "Understanding solid-phase microextraction: key factors influencing the extraction process and trends in improving the technique," Chem. Rev., vol. 113, pp. 1667-1685, Dec. 2012.

[10] A. Kloskowski, M. Pilarczyk, and J. Namieśnik, "Membrane solid-phase microextraction - a new concept of sorbent preparation," Anal. Chem., vol. 81, pp. 7363-7367, Aug. 2009.

[11] A. Spietelun, Ł. Marcinkowski, A. Kloskowski, and J. Namieśnik, "Determination of volatile organic compounds in water samples using Membrane-Solid Phase Microextraction (headspace version)," Analyst, vol. 138, pp. 5099-5106, May 2013.

[12] A. Spietelun, M. Pilarczyk, A. Kloskowski, and J. Namieśnik, "Current trends in solid-phase microextraction (SPME) fibre catings," Chem. Soc. Rev., vol. 39, pp. 4524-4537, Sep. 2010.

[13] E. Baltussen, P. Sandra, F. David, and C. Cramers, "Stir bar sorptive extraction (SBSE), a novel extraction technique for aqueous samples: Theory and principles," J. Microcolumn Sep., vol. 11, pp. 737-747, 1999.

[14] M. A. Jeannot and F. F. Cantwell, "Solvent microextraction into a single drop," Anal. Chem., vol. 68, pp. 2236-2240, July 1996.

[15] S. Pedersen-Bjergaard and K. E. Rasmussen, "Liquid-liquid-liquid microextraction for sample preparation of biological fluids prior to capillary electrophoresis," Anal. Chem., vol. 71, pp. 2650-2655, 1999.

[16] M. Rezaee, Y. Assadi, M. R. M. Hosseini, E. Aghaee, F. Ahmadi, and $\mathrm{S}$. Berijani, "Determination of organic compounds in water using dispersive liquid-liquid microextraction," J. Chromatogr. A, vol. 1116, pp. 1-9, May 2006.

Jacek Namieśnik was born in 1949. He obtained his Ph.D. in 1978 and has been a professor since 1996. He was the dean of the Chemical Faculty, Gdańsk University of Technology, from 1996 to 2002 and since 2005 he has been the head of the Department of Analytical Chemistry. He has also been the chairman of the Committee on Analytical Chemistry, Polish Academy of Sciences (PAS), since 2005. His major research interests include the development of new analytical procedures for determining trace and ultratrace constituents in samples with complex matrix compositions, the design and testing of customized analytical units and measuring devices, and the production of new types of matrix-free reference materials. He is the author and editor of 8 books and author and coauthor of over 300 papers, over 400 reports and communications published in conference proceedings, and 10 patents.

Agata Spietelun was born in 1984. She graduated from the Szczecin University of Technology with an M.Sc. degree in chemical technology in 2008, after which she started her Ph.D. studies at the Gdańsk University of Technology. Her research interests include the isolation and determination of analytes in environmental samples and extraction techniques in sample preparation, especially solid-phase microextraction.

Lukasz Marcinkowski was born in 1986. He graduated from the Gdańsk University of Technology with an M.Sc. degree in chemical technology in 2011. After that he started his Ph.D. studies at the Gdańsk University of Technology. His research interests include the physicochemical characterization of room temperature ionic liquids and their application in extraction techniques for determining polar organic compounds in environmental samples. 